\title{
Complexity of Concrete Type-Inference in the Presence of Exceptions ${ }^{*}$
}

\author{
Ramkrishna Chatterjee ${ }^{1}$ Barbara G. Ryder ${ }^{1}$ William A. Landi ${ }^{2}$ \\ 1 Department of Computer Science, Rutgers University, Piscataway, NJ 08855 USA, \\ Fax: 732445 0537, \{ramkrish,ryder\}@cs.rutgers.edu \\ 2 Siemens Corporate Research Inc, 755 College Rd. East, Princeton, NJ 08540 USA, \\ wlandi@scr.siemens.com
}

\begin{abstract}
Concrete type-inference for statically typed object-oriented programming languages (e.g., Java, $\mathrm{C}^{++}$) determines at each program point, those objects to which a reference may refer or a pointer may point during execution. A precise compile-time solution for this problem requires a flow-sensitive analysis. Our new complexity results for concrete type-inference distinguish the difficulty of the intraprocedural and interprocedural problem for languages with combinations of single-level types ${ }^{3}$, exceptions with or without subtyping, and dynamic dispatch. Our results include:

- The first polynomial-time algorithm for concrete type-inference in the presence of exceptions, which handles Java without threads, and $\mathrm{C}^{++}$;

- Proofs that the above algorithm is always safe and provably precise on programs with single-level types, exceptions without subtyping, and without dynamic dispatch;

- Proof that intraprocedural concrete type-inference problem with single-level types and exceptions with subtyping is PSPACEcomplete, while the interprocedural problem without dynamic dispatch is PSPACE-hard.
\end{abstract}

Other complexity characterizations of concrete type-inference for programs without exceptions are also presented.

\section{Introduction}

Concrete type-inference ( $C T I$ from now on) for statically typed object-oriented programming languages (e.g., Java, $\mathrm{C}^{++}$) determines at each program point, those objects to which a reference may refer or a pointer may point during execution. This information is crucial for static resolution of dynamically dispatched calls, side-effect analysis, testing, program slicing and aggressive compiler optimization.

\footnotetext{
* The research reported here was supported, in part, by NSF grant GER-9023628 and the Hewlett-Packard Corporation.

${ }^{3}$ These are types with data members only of primitive types.
} 
The problem of $C T I$ is both intraprocedurally and interprocedurally flowsensitive. However, there are approaches with varying degrees of flow-sensitivity for this problem. Although some of these have been used for pointer analysis of C, they can be adapted for CTI of Java without exceptions and threads, or $\mathrm{C}^{++}$without exceptions. At the one end of the spectrum are intraprocedurally and interprocedurally flow-insensitive approaches [Ste96, SH97, ZRL96, And94], which are the least expensive, but also the most imprecise. While at the other end are intraprocedurally and interprocedurally flow-sensitive approaches [LR92, EGH94, WL95, CBC93, MLR +93 , Ruf95], which are the most precise, but also the most expensive. Approaches like [PS91, PC94, Age95] are in between the above two extremes.

An intraprocedurally flow-insensitive algorithm does not distinguish between program points within a method; hence it reports the same solution for all program points within each method. In contrast, an intraprocedurally flow-sensitive algorithm tries to compute different solutions for distinct program points.

An interprocedurally flow-sensitive (i.e. context-sensitive) algorithm considers (sometimes approximately) only interprocedurally realizable paths [RHS95, LR91]: paths along which calls and returns are properly matched, while an interprocedurally flow-insensitive (i.e. context-insensitive) algorithm does not make this distinction. For the rest of this paper, we will use the term flow-sensitive to refer to an intra- and interprocedurally flow-sensitive analysis.

In this paper, we are interested in a flow-sensitive algorithm for $C T I$ of a robust subset of Java with exceptions, but without threads (this subset is described in Section 2). The complexity of flow-sensitive CTI in the presence of exceptions has not been studied previously. None of the previous flow-sensitive pointer analysis algorithms [LR92, WL95, EGH94, PR96, Ruf95, CBC93, MLR ${ }^{+93}$ for $\mathrm{C} / \mathrm{C}^{++}$handle exceptions. However, unlike in $\mathrm{C}^{++}$, exceptions are frequently used in Java programs, making it an important problem for Java.

The main contributions of this paper are:

- The first polynomial-time algorithm for $C T I$ in the presence of exceptions that handles a robust subset of Java without threads, and $\mathrm{C}^{++4}$,

- Proofs that the above algorithm is always safe and provably precise on programs with single-level types, exceptions without subtyping, and without dynamic dispatch; thus this case is in $\mathbf{P}$,

- Proof that intraprocedural $C T I$ for programs with single-level types and exceptions with subtyping is PSPACE-complete, while the interprocedural problem (even) without dynamic dispatch is PSPACE-hard.

- New complexity characterizations of $C T I$ in the absence of exceptions.

These results are summarized in table 1 , which also gives the sections of the paper containing these results.

The rest of this paper is organized as follows. First, we present a flow-sensitive algorithm, called the basic algorithm, for $C T I$ in the absence of exceptions, and discuss our results about complexity of $C T I$ in the absence of exceptions. Next,

\footnotetext{
${ }^{4}$ In this paper, we present our algorithm only for Java.
} 


\begin{tabular}{|c|c|c|c|c|c|}
\hline results & $\begin{array}{c}\text { paper } \\
\text { section }\end{array}$ & $\begin{array}{c}\text { single-level } \\
\text { types }\end{array}$ & $\begin{array}{c}\text { exceptions } \\
\text { without subtypes }\end{array}$ & $\begin{array}{c}\text { exceptions } \\
\text { with subtypes }\end{array}$ & $\begin{array}{c}\text { dynamic } \\
\text { dispatch }\end{array}$ \\
\hline $\begin{array}{c}\text { interprocedural CTI } \\
\text { in P, O }\left(n^{7}\right)\end{array}$ & $\sec 4$ & $\mathbf{x}$ & $\mathbf{x}$ & & \\
\hline $\begin{array}{c}\text { intraprocedural CTI } \\
\text { PSPACE-complete }\end{array}$ & $\operatorname{sec~4}$ & $\mathbf{x}$ & & $\mathbf{x}$ & \\
\hline $\begin{array}{c}\text { interprocedural CTI } \\
\text { PSPACE-hard }\end{array}$ & $\operatorname{sec~4}$ & $\mathbf{x}$ & & $\mathrm{x}$ & \\
\hline $\begin{array}{c}\text { interprocedural CTI } \\
\text { PSPACE-hard }\end{array}$ & $\operatorname{sec~3}$ & $\mathbf{x}$ & & & \\
\hline $\begin{array}{c}\text { interprocedural CTI } \\
\text { in P, O }\left(n^{5}\right)\end{array}$ & $\operatorname{sec~3}$ & $\mathrm{x}$ & & & \\
\hline $\begin{array}{c}\text { intraprocedural CTI } \\
\text { in NC }\end{array}$ & $\operatorname{sec~3}$ & $\mathrm{x}$ & & & \\
\hline
\end{tabular}

Table 1. Complexity results for $C T I$ summarized

we extend the basic algorithm for $C T I$ in the presence of exceptions, and discuss the complexity and correctness of the extended algorithm. Finally, we present PSPACE-hardness results about $C T I$ in the presence of exceptions. Due to lack of space, we have omitted all proofs. These proofs and further details about the results in this paper are given in [CRL97] ${ }^{5}$.

\section{Basic definitions}

Program representation. Our algorithm operates on an interprocedural control flow graph or ICFG [LR91]. An ICFG contains a control flow graph (CFG) for each method in the program. Each statement in a method is represented by a node in the method's CFG. Each call site is represented using a pair of nodes: a call-node and a return-node. Information flows from a call-node to the entrynode of a target method and comes back from the exit-node of the target method to the return-node of the call-node. Due to dynamic dispatch, interprocedural edges are constructed iteratively during data-flow analysis as in [EGH94]. Details of this construction are shown in Figure 3. We will denote the entry-node of main by start-node in the rest of this paper.

Representation of dynamically created objects. All run-time objects (or arrays) created at a program point $n$ are represented symbolically by object_n. No distinction is made between different elements of an array. Thus, if an array is created at $n$, object_n represents all elements of the array.

Precise solution for CTI. A reference variable is one of the following:

- a static variable (class variable) of reference ${ }^{6}$ type;

\footnotetext{
5 available at http://www.prolangs.rutgers.edu/refs/docs/tr341.ps.

${ }^{6}$ may refer to an instance of a class or an array.
} 
- a local variable of reference type;

- $A v$, where $A v$ is $V\left[t_{1}\right] \ldots\left[t_{d}\right]$, and

- $V$ is a static/local variable or $V$ is an array object $_{n}$ allocated at program point $n$, such that $V$ is either a $d$-dimensional array of reference type or an array of any type having more than $d$ dimensions and

- each $t_{i}$ is a non-negative integer; or

- $V \cdot s_{1} \ldots s_{k}$, where

- $V$ is either a static/local variable of reference type or $V$ is $A v$ or $V$ is

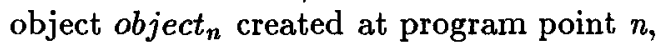

- for $1 \leq i \leq k$, each $V \cdot s_{1} \ldots s_{i-1}(V$ for $i=1)$ has the type of a reference to a class $T_{i}$ and each $s_{i}$ is a field of reference type of $T_{i}$ or $s_{i}=f_{i}\left[t_{i_{1}}\right] \ldots\left[t_{i_{r_{i}}}\right]$ and $f_{i}$ is a field of $T_{i}$ and $f_{i}$ is an array having at least $r_{i}$ dimensions and each $t_{i j}$ is a non-negative integer, and

- $V \cdot s_{1} \ldots s_{k}$ is of reference type.

Using these definitions, the precise solution for $C T I$ can be defined as follows: given a reference variable $R V$ and an object object_n, $\langle R V$, object_n $n\rangle$ belongs to the precise solution at a program point $n$ if and only if $R V$ is visible at $n$ and there exists an execution path from the start-node of the program to $n$ such that if this path is followed, $R V$ points to object_n at $n$ (i.e., at the top of $n$ ). Unfortunately, all paths in a program are not necessarily executable and determining which are executable is undecidable. Barth[Bar78] defined precise up to symbolic execution to be the precise solution under the assumption that all program paths are executable (i.e., the result of a test is independent of previous tests and all the branches are possible). In the rest of this paper we use precise to mean precise up to symbolic execution.

Points-to. A points-to has the form $\langle v a r, o b j\rangle$; where var is one of the following: (1) a static variable of reference type, (2) a local variable of reference type, (3) object_m - an array object created at program point $m$ or (4) object_n.f - field $f$, of reference type, of an object created at a program point $n$; and $o b j$ is object_s - an object created at a program point $s$.

Single-level type. A single-level type is one of the following: (1) a primitive type defined in [GJS96] (e.g., int, float etc.), (2) a class that has all non-static data-members of primitive types (e.g., class $A\{$ int $i, j ;\})$ or (3) an array of a primitive type.

Subtype. We use Java's definition of subtyping: a class $A$ is a subtype of another class $B$ if $A$ extends $B$, either directly or indirectly through inheritance.

Safe solution. An algorithm is said to compute a safe solution for CTI if and only if at each program point, the solution computed by the algorithm is a superset of the precise solution. 
Subset of Java considered. We essentially consider a subset that excludes threads, but in some cases we may need to exclude three other features: finalize methods, static initializations and dynamically defined classes. Since finalize methods are called (non-deterministically) during garbage collection or unloading of classes, if a finalize method modifies a variable of reference type (extremely rare), it cannot be handled by our algorithm. Static initializations complicate analysis due to dynamic loading of classes. If static initializations can be done in program order, our algorithm can handle them. Otherwise, if they depend upon dynamic loading (extremely rare), our algorithm cannot handle them. Similarly, our algorithm cannot handle classes that are constructed on the fly and not known statically. We will refer to this subset as JavaWoThreads.

Also, we have considered only exceptions generated by throw statements. Since run-time exceptions can be generated by almost any statement, we have ignored them. Our algorithm can handle run-time exceptions if the set of statements that can generate these exceptions is given as an input. If all statements that can potentially generate run-time exceptions are considered, we will get a safe solution; however, this may generate far more information than what is useful.

\section{$3 \quad C T I$ in the absence of exceptions}

Our basic algorithm for $C T I$ is an iterative worklist algorithm [KU76]. It operates on an ICFG and is similar to the Landi-Ryder algorithm [LR92] for alias analysis, but instead of aliases, it computes points-tos. In Section 4, we will extend this algorithm to handle exceptions.

Lattice for data-flow analysis. In order to restrict data-flow only to realizable paths, points-tos are computed conditioned on assumed-points-tos (akin to reaching alias in [LR92] [PR96]), which represent points-tos reaching the entry of a method, and approximate the calling context in which the method has been called (see the example in Appendix A). A points-to along with its assumedpoints-to is called a conditional-points-to. A conditional-points-to has the form 〈condition, points-to), where condition is an assumed-points-to or empty (meaning this points-to is applicable to all contexts). For simplicity, we will write $\langle$ empty,points-to as points-to. Also a special data-flow element reachable is used to check whether a node is reachable from the start-node through a realizable path. This ensures that only such reachable nodes are considered during dataflow analysis and only points-tos generated by them are put on the worklist for propagation. The lattice for data-flow analysis (associated with a program point) is a subset lattice consisting of sets of such conditional-points-tos and the data-flow element reachable.

Query. Using these conditional-points-tos, a query for $C T I$ is answered as follows. Given a reference variable $V$ and a program point $l$, the conditionalpoints-tos with compatible assumed-points-tos computed at $l$ are combined to 
determine the possible values of $V$. Assumed-points-tos are compatible if and only if they do not imply different values for the same user defined variable. For example, if $V$ is $p . f 1$, and the solution computed at $l$ contains $\langle e m p t y,\langle p, o b j 1\rangle\rangle$, $\langle\mathrm{z},\langle o b j 1 . f 1, o b j 2\rangle\rangle$ and $\langle\mathrm{u},\langle o b j 1 . f 1, o b j 3\rangle\rangle$, then the possible values of $V$ are obj2 and $o b j 3$.

Algorithm description. Figure 1 contains a high-level description of the main loop of the basic algorithm. apply computes the effect of a statement on an incoming conditional-points-to. For example, suppose $l$ labels the statement $p . f 1$ $=q, n d f_{-} e l m$ (i.e. the points-to reaching the top of $l$ ) is $\langle z,\langle p$, object_s $\rangle\rangle$ and $\langle u,\langle q$, object_n $n\rangle$ is present in the solution computed at $l$ so far. Assuming $z$ and $u$ are compatible, apply generates $\langle$ object_s.f1, object_n〉 under the condition that both $z$ and $u$ hold at the entry-node of the method containing $l$. Then either $z$ or $u$ is chosen as the condition for the generated data-flow element. For example, if $u$ is chosen then $\langle u,\langle$ object_s.f1, object_n $n\rangle$ will be generated. When a conjunction of conditions is associated with a points-to, any fixed-size subset of these conditions may be stored without affecting safety. At a program point where this data-flow element is used, if all the conjuncts are true then any subset of the conjuncts is also true. This may cause overestimation of solution at program points where only a proper subset of the conjuncts is true. At present, we store only the first member of the list of conditions. apply is defined in Appendix B.

add_to_solution_and_worklist_if_needed checks whether a data-flow element is present in the solution set (computed so far) of a node. If not, it adds the dataflow element to the solution set, and puts the node along with this data-flow element on the worklist.

process_exit_node propagates data-flow elements from the exit-node of a method to the return-node of a call site of this method. Suppose $\langle z, u\rangle$ holds at the exit-node of a method $M$. Consider a return-node $R$ of a call site $C$ of $M$. For each assumed-points-to $x$ such that $\langle x, t\rangle$ is in the solution set at $C$ and $t$ implies $z$ at the entry-node of $M,\langle x, u\rangle$ is propagated by process_exit_node to $R$. process_exit_node is defined in Figure 2.

process_call_node propagates data-flow elements from a call site to the entrynode of a method called from this site. Due to dynamic dispatch, the set of methods invoked from a call site is iteratively computed during the data-flow analysis as in [EGH94]. Suppose $\langle x, t\rangle$ holds at a call site $C$ which has a method $M$ in its set of invocable methods computed so far. If $t$ implies a points-to $z$ at the entry-node of $M$ (e.g., through an actual to formal binding), $\langle z, z\rangle$ is forwarded to the entry-node of $M$. process_call_node also remembers the association between $x$ and $z$ at $C$ because this is used by process_exit_node as described above. process_call_node is defined in Figures 3 and 4.

Other functions used by the above routines are defined in Appendix B. Appendix A contains an example which illustrates the basic algorithm.

Precision of the basic algorithm. By induction on the number of iterations needed to compute a data-flow element and the length of a path associated with a data-flow element, in [CRL97], we prove that the basic algorithm computes the 


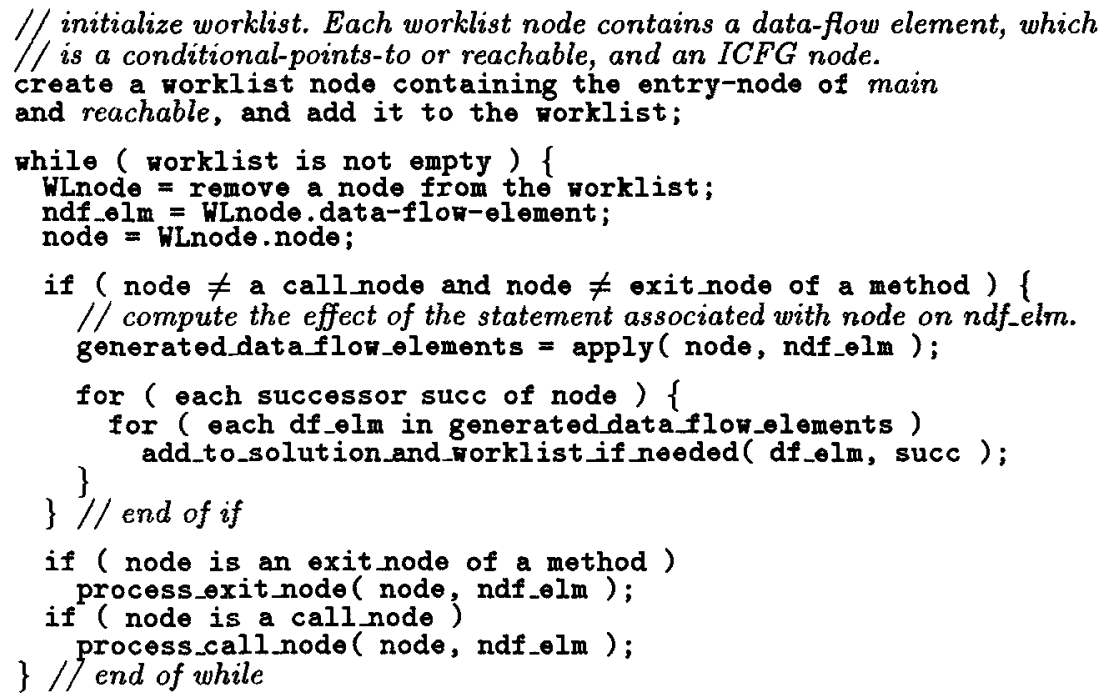

Fig. 1. High-level description of the basic algorithm

precise solution for programs with only single-level types and without dynamic dispatch, exceptions or threads. For programs of this form, CTI is distributive and a conditional-points-to at a program point can never require the simultaneous occurrence of multiple conditional-points-tos at (any of) its predecessors. Intuitively this is why the above proof works. The presence of general types, dynamically dispatched calls or exceptions with subtyping violate this condition and hence $C T I$ is not polynomial-time solvable in the presence of these constructs. We also prove that the basic algorithm computes a safe solution for programs written in JavaWoThreads, but without exceptions.

Complexity of the basic algorithm. The complexity of the basic algorithm for programs with only single-level types and without dynamic dispatch, exceptions or threads is $O\left(n^{5}\right)$, where $n$ is approximately the number of statements in the input program. This an improvement over the $O\left(n^{7}\right)$ worst-case bound achievable by applying previous approaches of [RHS95] and [LR91] to this case. Note that $\mathrm{O}\left(n^{3}\right)$ is a trivial worst-case lower bound for obtaining a precise solution for this case. For programs written in JavaWoThreads, but without exceptions, the basic algorithm is polynomial-time.

Other results on the complexity of $C T I$ in the absence of exceptions. In [CRL97], we prove the following two theorems:

Theorem 1 Intraprocedural CTI for programs with only single-level types is in non-deterministic log-space and hence NC. 


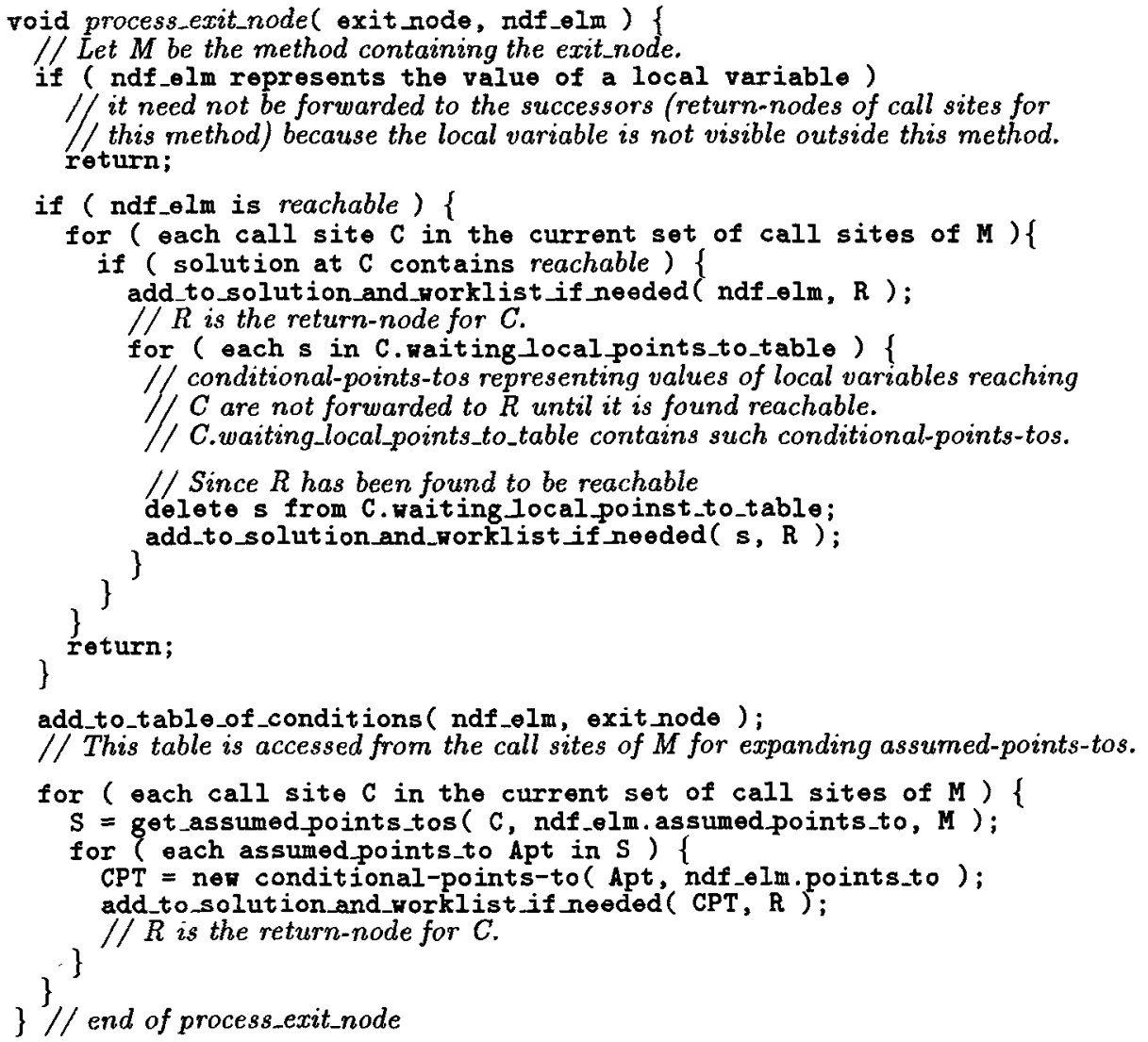

Fig. 2. Code for processing an exit-node

Recall that non-deterministic log-space is the set of languages accepted by nondeterministic Turing machines using logarithmic space[Pap94] and NC is the class of efficiently parallelizable problems which contains non-deterministic logspace.

\section{Theorem 2}

CTI for programs with only single-level types and dynamic dispatch is PSPACEhard.

\section{Algorithm for $C T I$ in the presence of exceptions}

In this section we extend the basic algorithm for CTI of JavaWoThreads, and discuss the complexity and precision of this extended algorithm.

Data-flow Elements: The data-flow elements propagated by this extended algorithm have one of the following forms: 


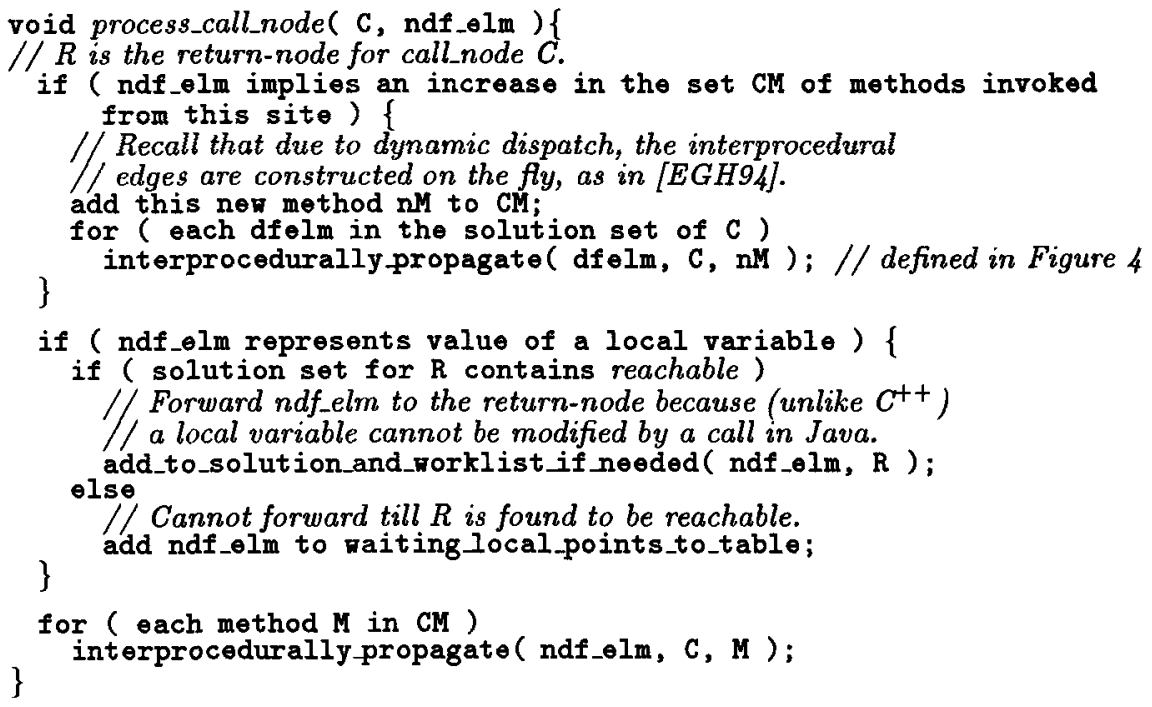

Fig. 3. Code for processing a call-node

1. 〈reachable ,

2. 〈label, reachable $\rangle$,

3. $\langle$ excp-type, reachable $\rangle$,

4. $\langle z, u\rangle$,

5. $\langle l a b e l, z, u\rangle$,

6. $\langle$ excp-type, $z, u\rangle$,

7. $\langle e x c p, z, o b j\rangle$.

Here $z$ and $u$ are points-tos. The lattice for data-flow analysis associated with a program point is a subset lattice consisting of sets of these data-flow elements. In the rest of this section, we present definitions of these data-flow elements and a brief description of how they are propagated. Further details are given in [CRL97]. First we describe how a throw statement is handled. Next, we describe propagation at a method exit-node. Finally, we describe how a finally statement is handled.

throw statement: In addition to the conditional-points-tos described previously, this algorithm uses another kind of conditional-points-tos, called exceptionalconditional-points-tos, which capture propagation due to exceptions. The conditional part of these points-tos consists of an exception type and an assumed points-to (as before). Consider a throw statement $l$ in a method Proc, which throws an object of type $\mathrm{T}$ (run-time type and not the declared type). Moreover let $\langle\langle q, o b j 1\rangle,\langle p, o b j 2\rangle\rangle$ be a conditional-points-to reaching the top of $l$. At the throw statement, this points-to is transformed to $\langle T,\langle q, o b j 1\rangle,\langle p, o b j 2\rangle\rangle$ and 


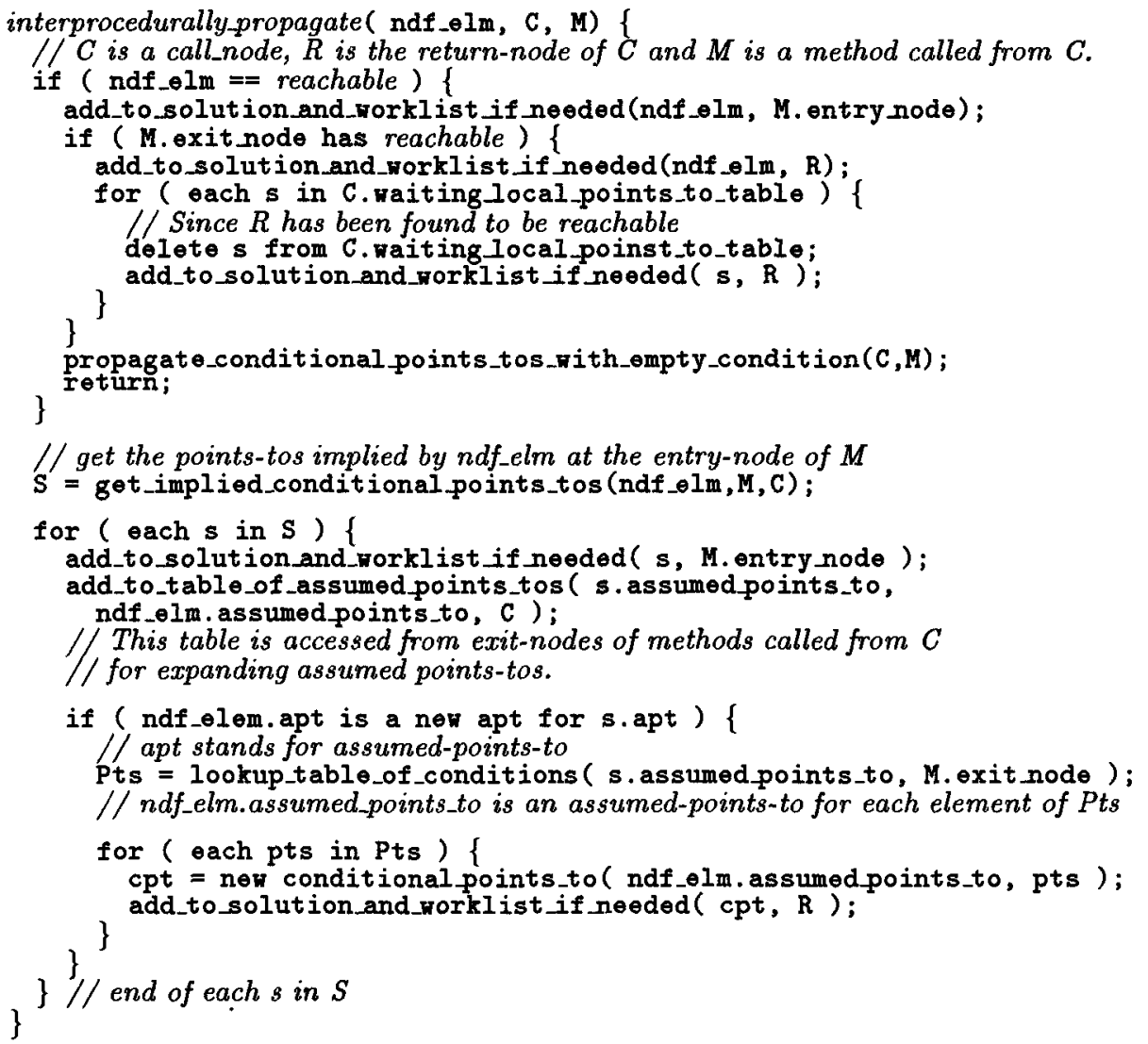

Fig. 4. Code for interprocedurally_propagate

propagated to the exit-node of the corresponding try statement, if there is one. A precalculated catch-table at this node is checked to see if this exception (identified by its type $T$ ) can be caught by any of the corresponding catch statements. If so, this exceptional-conditional-points-to is forwarded to the entry-node of this catch statement, where it is changed back into an ordinary conditional-points-to $\langle\langle q, o b j 1\rangle,\langle p, o b j 2\rangle\rangle$. If not, this exceptional-conditional-points-to is forwarded to the entry-node of a finally statement (if any), or the exit-node of the innermost enclosing try, catch, finally or the method body.

A throw statement also generates a data-flow element for the exception itself. Suppose the thrown object is $o b j$ and it is the thrown object under the assumed points-to $\langle p, o b j 1\rangle$. Then $\langle e x c p,\langle p, o b j 1\rangle, o b j\rangle$ representing the exception is generated. Such data-flow elements are handled like exceptional-conditional-pointstos, described above. If such a data-flow element reaches the entry of a catch statement, it is used to instantiate the parameter of the catch statement. 
In addition to propagating reachable (defined in section 3), this algorithm also propagates data-flow elements of the form 〈excp-type, reachable $\rangle$. When $\langle$ reachable reaches a throw statement, it is transformed into 〈excp-type, reachable , where excp-type is a run-time type of the exception thrown, which is then propagated like other exceptional-conditional-points-tos.

If the throw is not directly contained in a try statement, then the dataflow elements generated by it are propagated to the exit-node of the innermost enclosing catch, finally or method body.

exit-node of a method: At the exit-node of a method, a data-flow element of type 4,6 or 7 is forwarded (after replacing the assumed points-to as described in section 3) to the return-node of a call site of this method if and only if the assumed points-to of the data-flow element holds at the call site. At a returnnode, ordinary conditional-points-tos (type 4) are handled as before. However, a data-flow element of type 6 or 7 is handled as if it were generated by a throw at this return-node.

finally statement: The semantics of exception handling in Java is more complicated than other languages like $\mathrm{C}^{++}$because of the finally statement. A try statement can optionally have a finally statement associated with it. It is executed no matter how the try statement terminates: normally or due to an exception. A finally statement is always entered with a reason, which could be an exception thrown in the corresponding try statement or one of the corresponding catch statements, or leaving the try statement or one of its catch clauses by a return, (labelled) break or (labelled) continue, or by falling through. This reason is remembered on entering a finally, and unless the finally statement itself creates its own reason to exit the finally, at the exit-node of the finally this reason is used to decide control flow. If the finally itself creates its own reason to exit itself (e.g., due to an exception), then this new reason overrides any previous reason for entering the finally. Also, nested finally statements cause reasons for entering them to stack up. In order to correctly handle this involved semantics, for all data-flow elements entering a finally, the algorithm remembers the reason for entering it. For data-flow elements of type 3,6 or 7 (enumerated above), the associated exception already represents this reason. A label is associated with data-flow elements of type 1 or 4, which represents the statement number to which control should go after exit from the finally. Thus the data-flow elements in a finally have one of the following forms:

1. 〈label, reachable ,

2. 〈excp-type, reachable $\rangle$,

3. $\langle$ label, $z, u\rangle$,

4. $\langle$ excp-type, $z, u\rangle$,

5. $\langle e x c p, z, o b j\rangle$.

When a labelled data-flow element reaches the labelled statement, the label is dropped and it is transformed into the corresponding unlabelled data-flow element. 
Inside a finally, due to labels and exception types associated with data-flow elements, apply uses a different criterion for combining data-flow elements (at an assignment node) than the one given in section 3. Two data-flow elements $\langle x 1, y 1, z 1\rangle$ and $\langle x 2, y 2, z 2\rangle$ can be combined if and only if both $x 1$ and $x 2$ represent the same exception type or the same label, and $y 1$ and $y 2$ are compatible (as defined in section 3 ).

At a call statement (inside a finally), if a data-flow element has a label or an exception type associated with it, it is treated as part of the context (assumed points-to) and not forwarded to the target node. It is put back when assumed points-tos are expanded at an exit-node of a method. For exceptionalconditional-points-tos or data-flow elements representing exceptions, the exceptions associated with them at the exit-node override any label or exception type associated with their assumed points-tos at a corresponding call site. Data-flow

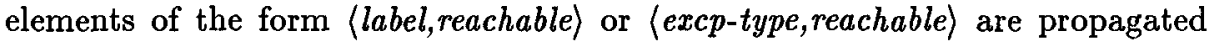
across a call if and only if $\langle$ reachable $\rangle$ reaches the exit-node of one of the called methods. A mechanism similar to the one used for handling a call is used for handling a try statement nested inside a finally because it can cause labels and exceptions to stack up. Details of this are given in [CRL97].

If the finally generates a reason of its own for exiting itself, the previous label/exception-type associated with a data-flow element is discarded, and the new label/exception-type representing this reason for leaving the finally is associated with the data-flow element.

Example. The example in Figure 5 illustrates the above algorithm.

Precision of the extended algorithm. In [CRL97], we prove that the extended algorithm described in Section 4 computes the precise solution for programs with only single-level types, exceptions without subtyping, and without dynamic dispatch. We also prove that this algorithm computes a safe solution for programs written in JavaWoThreads.

Complexity of the extended algorithm. The the worst-case complexity of the extended algorithm for programs with only single-level types, exceptions without subtyping, and without dynamic dispatch is $\mathrm{O}\left(n^{7}\right)$. Since we have proved that the algorithm is precise for this case, this shows that this case is in $P$. If we disallow trys nested inside a finally, the worst-case complexity is $\mathrm{O}\left(n^{6}\right)$. For general programs written in JavaWoThreads, the extended algorithm is polynomialtime.

Complexity due to exceptions with subtyping. In [CRL97], we prove the following theorem:

Theorem 3 Intraprocedural CTI for programs with only single-level types and exceptions with subtyping is PSPACE-complete; while the interprocedural case (even) without dynamic dispatch is PSPACE-hard. 


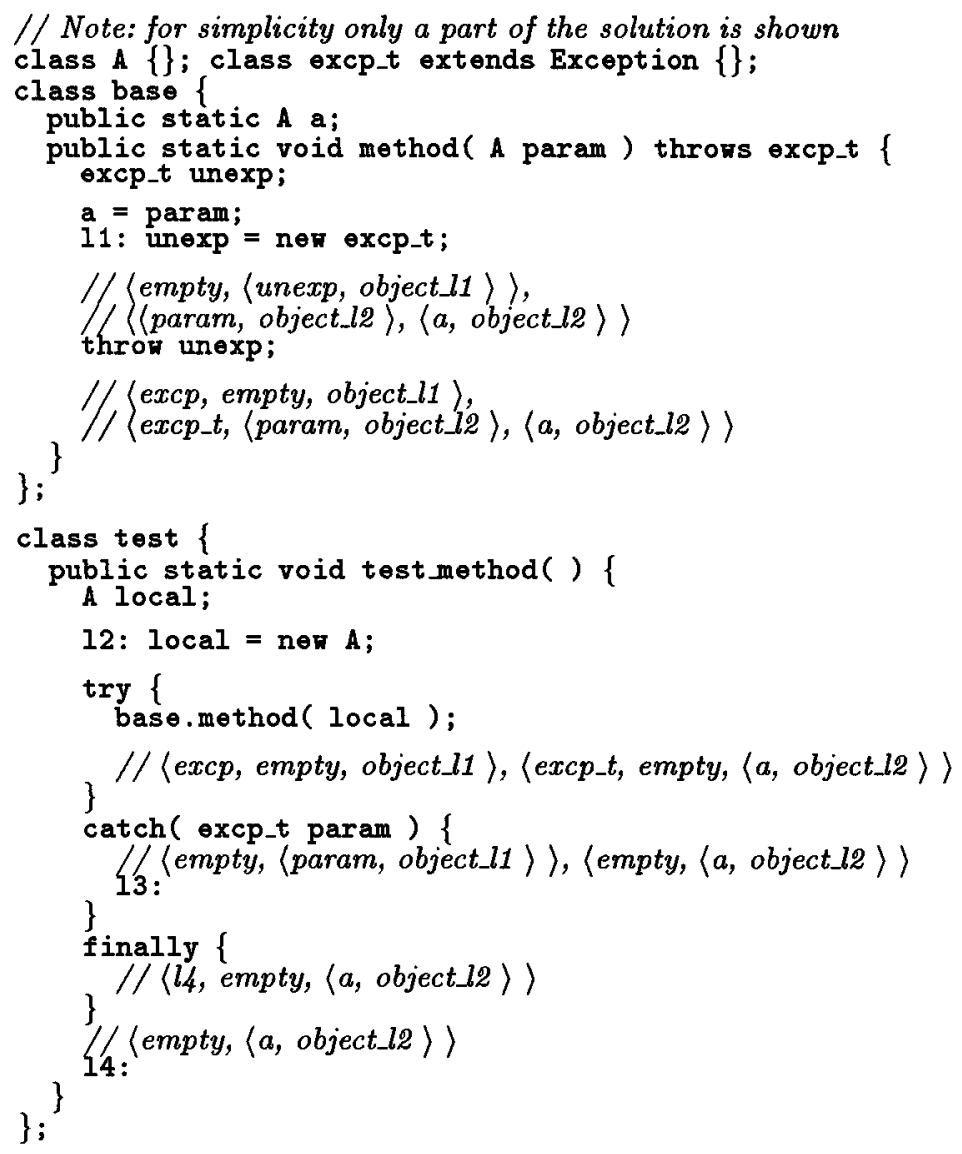

Fig. 5. $C T I$ in the presence of exceptions

Theorems 2 and 3 show that in the presence of exceptions, among all the reasonable special cases that we have considered, programs with only single-level types, exceptions without subtyping, and without dynamic dispatch comprise the only natural special case that is in P. Note that just adding subtyping for exception types and allowing overloaded catch clauses increase complexity from $P$ to PSPACE-hard.

\section{Related work}

As mentioned in the introduction, no previous algorithm for pointer analysis or $C T I$ handles exceptions. This work takes state-of-the-art in pointer analysis one step further by handling exceptions. Our algorithm differs from other pointer analysis and CTI algorithms [EGH94, WL95, Ruf95, PC94, PS91, CBC93, 
MLR+93] in the way it maintains context-sensitivity by associating assumedpoints-tos with each data-flow element, rather than using some approximation of the call stack. This way of handling context-sensitivity enables us to obtain precise solution for polynomial-time solvable cases, and handle exceptions. This way of maintaining context is similar to Landi-Ryder's[LR92] method of storing context using reaching aliases, except that our algorithm uses points-tos rather than aliases. Our algorithm also differs from approaches like [PS91, Age95] in being intraprocedurally flow-sensitive.

\section{Conclusion}

In this paper, we have studied the complexity $C T I$ for a subset of Java, which includes exceptions. To the best of our knowledge, the complexity of $C T I$ in the presence of exceptions has not been studied before. The following are the main contributions of this work (proofs are not presented in this paper, but appear in [CRL97]):

1. The first polynomial-time algorithm for $C T I$ in the presence of exceptions which handles a robust subset of Java without threads, and $\mathrm{C}^{++}$.

2. A proof that $C T I$ for programs with only single-level types, exceptions without subtyping, and without dynamic dispatch is in $\mathbf{P}$ and can be solved in $\mathrm{O}\left(n^{7}\right)$ time.

3. A proof that intraprocedural $C T I$ for programs with only single-level types, exceptions with subtyping, and without dynamic dispatch is PSPACEcomplete, and the interprocedural case is PSPACE-hard.

Additional contributions are:

1. A proof that $C T I$ for programs with only single-level types, dynamic dispatch, and without exceptions is PSPACE-hard.

2. A proof that $C T I$ for programs with only single-level types can be done in $\mathrm{O}\left(n^{5}\right)$ time. This is an improvement over the $\mathrm{O}\left(n^{7}\right)$ worst-case bound achievable by applying previous approaches of [RHS95] and [LR91] to this case.

3. A proof that intraprocedural $C T I$ for programs with only single-level types is in non-deterministic log-space and hence $N C$.

\section{References}

[Age95] Ole Agesen. The cartesian product algorithm: Simple and precise type inference of parametric polymorphism. In Proceedings of European Conference on Object-oriented Programming (ECOOP '95), 1995.

[And94] L. O. Andersen. Program Analysis and Specialization for the C Programming Language. PhD thesis, DIKU, University of Copenhagen, 1994. Also available as DIKU report $94 / 19$. 
[Bar78] J. M. Barth. A practical interprocedural data flow analysis algorithm. Communications of the ACM, 21(9):724-736, 1978.

[CBC93] Jong-Deok Choi, Michael Burke, and Paul Carini. Efficient flow-sensitive interprocedural computation of pointer-induced aliases and side effects. In Proceedings of the ACM SIGPLAN/SIGACT Symposium on Principles of Programming Languages, pages 232-245, January 1993.

[CRL97] Ramkrishna Chatterjee, Barbara Ryder, and William Landi. Complexity of concrete type-inference in the presence of exceptions. Technical Report DCS-TR-341, Dept of CS, Rutgers University, September 1997.

[EGH94] Maryam Emami, Rakesh Ghiya, and Laurie J. Hendren. Context-sensitive interprocedural points-to analysis in the presence of function pointers. In Proceedings of the ACM SIGPLAN Conference on Programming language design and implementation, pages 242-256, 1994.

[GJS96] James Gosling, Bill Joy, and Guy Steele. The Java Language Specification. Addison-Wesley, 1996.

[KU76] J.B. Kam and J.D. Ullman. Global data flow analysis and iterative algorithms. Journal of $A C M, 23(1): 158-171,1976$.

[LR91] W.A. Landi and Barbara G. Ryder. Pointer-induced aliasing: A problem classification. In Proceedings of the ACM SIGPLAN/SIGACT Symposium on Principles of Programming Languages, pages 93-103, January 1991.

[LR92] W.A. Landi and Barbara G. Ryder. A safe approximation algorithm for interprocedural pointer aliasing. In Proceedings of the ACM SIGPLAN Conference on Programming Language Design and Implementation, pages 235-248, June 1992.

$\left[\mathrm{MLR}^{+}\right.$93] T. J. Marlowe, W. A. Landi, B. G. Ryder, J. Choi, M. Burke, and P. Carini. Pointer-induced aliasing: A clarification. ACM SIGPLAN Notices, 28(9):67-70, September 1993.

[Pap94] C. H. Papadimitriou. Computational Complexity. Addison-Wesley, 1994.

[PC94] J. Plevyak and A. Chien. Precise concrete type inference for object oriented languages. In Proceeding of Conference on Object-Oriented Programming Systems, Languages and Applications (OOPSLA '94), pages 324-340, October 1994.

[PR96] Hemant Pande and Barbara G. Ryder. Data-flow-based virtual function resolution. In LNCS 1145, Proceedings of the Third International Symposium on Static Analysis, 1996.

[PS91] J. Palsberg and M. Schwartzbach. Object-oriented type inference. In Proceedings of Conference on Object-Oriented Programming Systems, Languages, and Applications (OOPSLA'91), pages 146-161, October 1991.

[RHS95] T. Reps, S. Horwitz, and M. Sagiv. Precise interprocedural dataflow analysis via graph reachability. In Proceedings of the ACM SIGPLAN/SIGACT Symposium on Principles of Programming Languages, pages 49-61, 1995.

[Ruf95] E. Ruf. Context-insensitive alias analysis reconsidered. In Proceedings of the ACM SIGPLAN Conference on Programming language design and implementation, pages 13-22, June 1995.

[SH97] M. Shapiro and S. Horwitz. Fast and accurate flow-insensitive points-to analysis. In Proceedings of the ACM SIGPLAN/SIGACT Symposium on Principles of Programming Languages, pages 1-14, 1997.

[Ste96] Bjarne Steensgaard. Points-to analysis in almost linear time. In Proceedings of the ACM SIGPLAN/SIGACT Symposium on Principles of Programming Languages, pages 32-41, 1996. 
[WL95] Robert P. Wilson and Monica S. Lam. Efficient context-sensitive pointer analysis for c programs. In Proceedings of the ACM SIGPLAN Conference on Programming language design and implementation, pages 1-12, 1995.

[ZRL96] S. Zhang, B. G. Ryder, and W. Landi. Program decomposition for pointer aliasing: A step towards practical analyses. In Proceedings of the 4 th Symposium on the Foundations of Software Engineering, October 1996.

\section{A Example for the basic algorithm}

// Note: due to lack of space only a part of the solution is shown

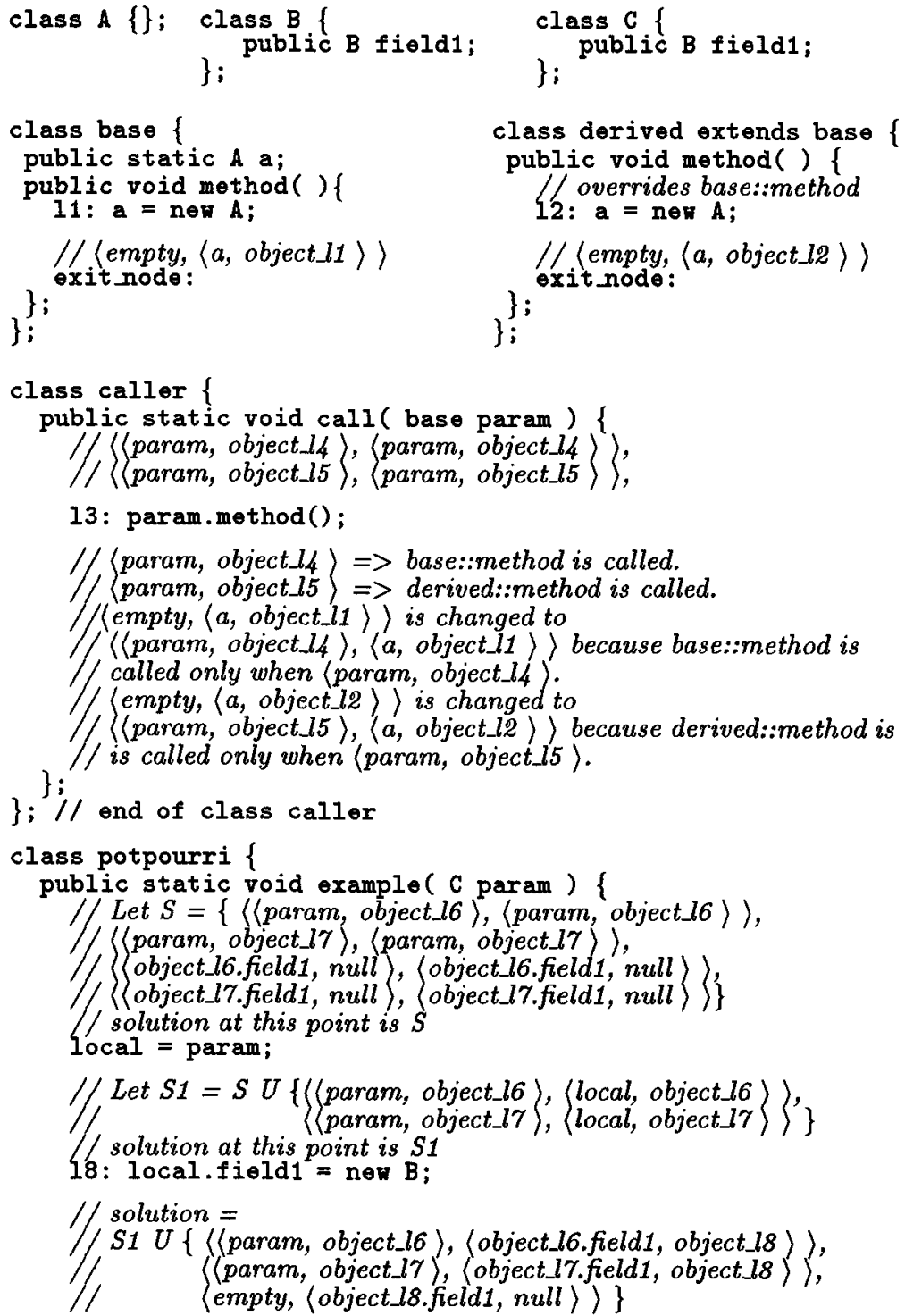




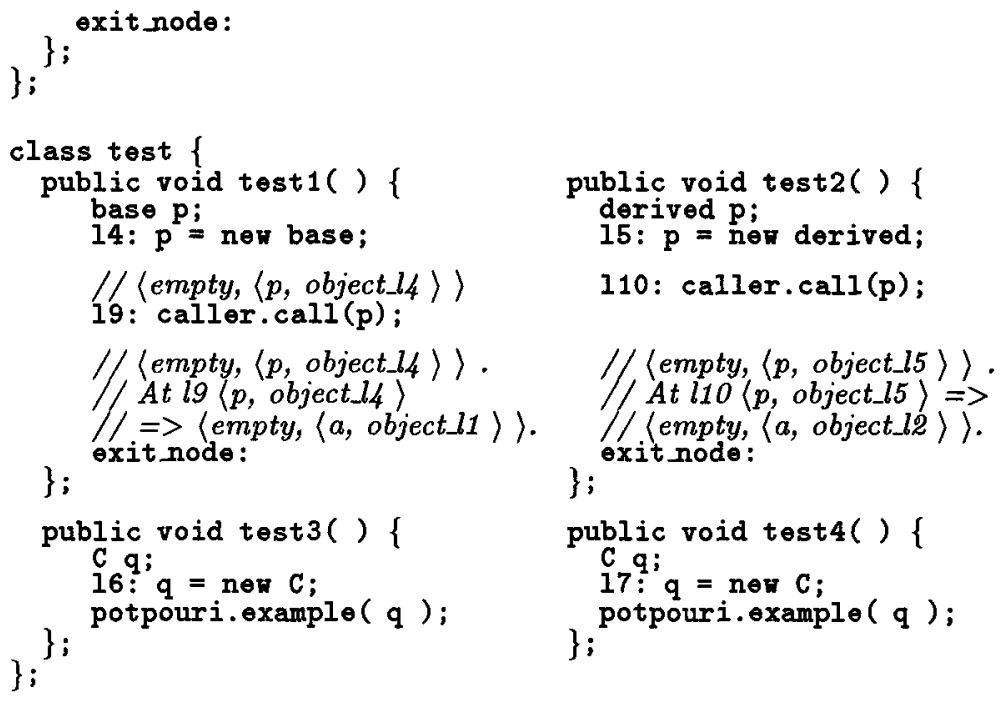

\section{B Auxiliary functions}

// CPT stands for a conditional-points-to. DFE stands for a

/ data-flow-element which could be a CPT or 'reachable'.

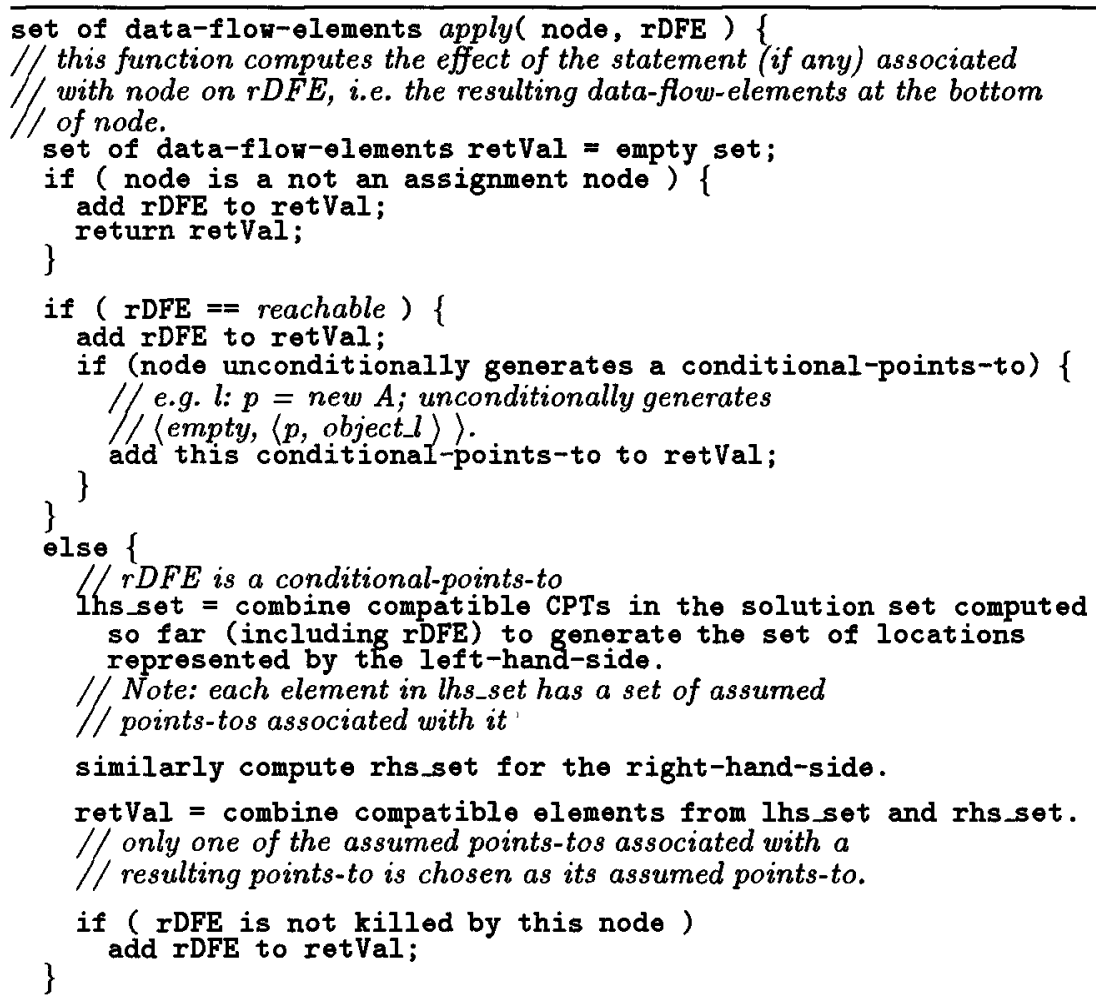


return retVal;

\} // end of apply

void add_to_table_of_conditions( ICPT, exit-node) (

// each exit-node has a table condTable associated with it,

/ which stores for each assumed points-to, the points-tos

/ which hold at the exit-node with this assumed points-to.

/ This function stores rCPT in this table. \}

void add_to_table_of_assumed_points_tos(s, condition, c) \{

$/ C$ is a call-node, condition is an assumed-points-to and $s$ is a

/ points-to passed to the entry-node of a method invoked from $C$.

/ Each call-node has a table asPtTtable associated with it, which

/ stores for each points-to that is passed to the entry-node of a

/ method invoked from this site, the assumed-points-tos which

/ imply this point-to at the call site. This function stores

/ condition with $s$ in this table. \}

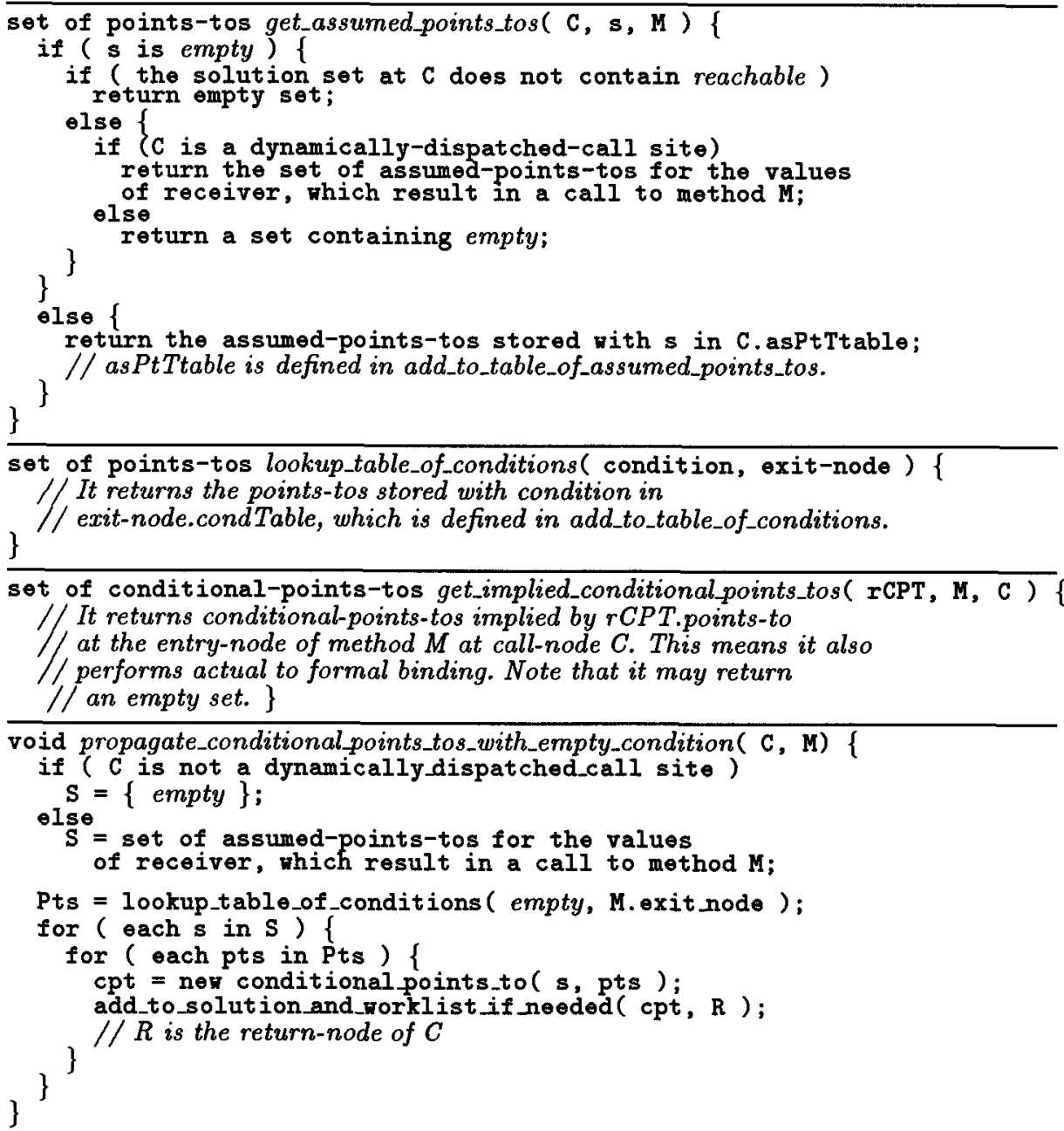

